\title{
Desensitization of Triggers and Urge Reduction for Paruresis: A Case Report
}

\author{
Hyoin Park ${ }^{1}$, Daeho Kim ${ }^{1 凶}$, Eun Young Jang ${ }^{1}$, and Hwallip Bae ${ }^{2}$ \\ ${ }^{1}$ Department of Psychiatry and Mental Health Research Institute, Hanyang University College of Medicine, Seoul, Republic of Korea \\ ${ }^{2}$ Department of Psychiatry, Myongji Hospital, Goyang, Republic of Korea
}

Paruresis is a special type of non-generalized social phobia that involves fear and avoidance of urination in public restrooms. We administered eight 60-minute sessions of desensitization of triggers and urge reduction (DeTUR), an addiction protocol of eye movement desensitization and reprocessing (EMDR) therapy, to a 29-year old man with paruresis of 10 year duration. Because phobic avoidance is the hallmark of any anxiety disorder, we applied DeTUR targeting the urge to avoid each anxiety-provoking situation in succession. After treatment, the participant no longer met the requirements for a diagnosis of social anxiety disorder, and the self-reported symptoms of social anxiety had decreased to non-clinical levels; furthermore, these treatment gains were maintained at the one-year follow-up. Further clinical studies are needed to generalize this finding.

Psychiatry Investig 2016;13(1):161-163

Key Words Paruresis, Social phobia, Social anxiety disorder, Eye movement desensitization and reprocessing therapy, Desensitization of triggers and urge reduction, Treatment.

\section{INTRODUCTION}

Paruresis, also known as shy bladder syndrome is a specialized form of social anxiety disorder that involves fear and avoidance of urination in the presence of others such as in public facilities. ${ }^{1}$ There has been little research on the treatment of this disorder. Although no controlled studies have been conducted, cognitive-behavioral and pharmacological interventions have been tried and described in case studies. ${ }^{2-4}$ Eye movement desensitization and reprocessing (EMDR) is an established psychological treatment for post-traumatic stress disorder. It has also been applied to anxiety disorders such as specific phobias, panic disorder, and generalized anxiety disorder but not to social anxiety disorder. ${ }^{5-7}$ These previous studies used the standard trauma protocol of EMDR although the targets, (i.e., traumatic memories) were replaced by precipitating events or experiences of anxiety symptoms.

Received: January 30, 2015 Revised: May 8, 2015

Accepted: May 8, 2015 Available online: November 20, 2015

$\triangle$ Correspondence: Daeho Kim, MD, PhD

Department of Psychiatry, Hanyang University Medical School, 222 Wangsimni-ro, Seongdong-gu, Seoul 04763, Republic of Korea

Tel: +82-2-2290-8430, Fax: +82-2-2298-2055

E-mail: dkim9289@hanyang.ac.kr

(a) This is an Open Access article distributed under the terms of the Creative Commons Attribution Non-Commercial License (http://creativecommons.org/licenses/by$\mathrm{nc} / 3.0$ ) which permits unrestricted non-commercial use, distribution, and reproduction in any medium, provided the original work is properly cited.
Desensitization of triggers and urge reprocessing (DeTUR) was originally developed to treat urge-related conditions such as chemical dependency and behavioral addiction; like EMDR, it involves brief exposures to targets, and spontaneous association is repeated along with bilateral stimulation. However, procedurally, it is different from EMDR in that it is aimed at triggers of craving or situations involving addictive substances or behaviors, and it deals only with urges (level of urge, LOU) and body sensations rather than images, cognition, emotions, and body sensations evoked by traumatic memories, as in standard EMDR. ${ }^{8}$ A hierarchy of triggers involving progressive levels of urge is established, and each trigger is processed along with a bilateral stimulus such as horizontal eye movements or alternative tactile stimuli, until the urge becomes neutral or disappears.

There has been no previous study using DeTUR in the treatment of anxiety disorder; however, this approach appears suitable because strategic avoidance is the main psychopathology of social anxiety ${ }^{9}$ and can be expressed in terms of the urge to avoid a situation, and DeTUR has components similar to gradual imaginal exposure. We therefore sought to test the efficacy of DeTUR with the urge to engage in addictive behaviors replaced by the urge to avoid anxiety-provoking situations. 


\section{CASE}

A 27 year-old man visited our outpatient clinic in the Department of Psychiatry, Hanyang University Guri Hospital in Gyeonggi Province, Korea, complaining of fear of using public restrooms and worrying about others watching him urinate. This fear had begun 10 years earlier when he was a sophomore in high school; since then, he had persistently avoided such places, which made it difficult for him to travel or engage in social activities. He managed to enter and use the restroom if only one or two others were in it, but could not use a restroom with several other people in it. This condition had greatly impaired his academic progress and interpersonal activities. During the course of his illness, he had attended a psychiatric clinic and received a prescription for antidepressants and antianxiety medications, but there had been no improvement over one year of use. In addition, he had attended six sessions of individual cognitive behavioral therapy for social phobia but had terminated the treatment prematurely due to a lack of perceived improvement. He had heard out about EMDR from the media and visited our clinic with the hope receiving the treatment.

The patient met the DSM-5 criteria for social anxiety disorder in that he had consistently experienced marked fear and anxiety about using public restrooms while others were present, and he feared that other people would notice his anxiety and embarrassment.

He was referred to EMDR therapy with the first author (HP) and privoded informed consent for receiving DeTUR and participating in a case report. One week before initiation of the DeTUR, his baseline symptom level was measured with the State Anxiety Inventory (SAI), ${ }^{10}$ the Social Avoidance and Distress Scale (SAD), ${ }^{11}$ and the Fear of Negative Evaluation (FNE). ${ }^{11}$ He completed the questionnaires again one week after the final session and at the one-year follow-up. The patient attended eight weekly 60-minute sessions, at the end of which he had no anxiety about using any public restroom, and his self-reported symptoms on the three measures had reached nonclin- ical levels. State anxiety (score range 20-80, cut-off 52) decreased from 56 pretreatment to 39 post-treatment and 26 after one year. Social avoidance and distress (range 28-140, cutoff 61) decreased from 98 pretreatment to 53 post-treatment and 44 after one year. Fear of negative evaluation decreased from 48 pretreatment to 21 post-treatment and 26 after one year (range 12-60, cut-off 42).

In the first session, the patient was given information about EMDR therapy, a brief history was taken, and he was taught about a safe place exercise: guided imagery for controlling possible distress between sessions. The therapist encouraged him to use this image when he experienced distress outside the sessions. His second session began the actual DeTUR protocol, and the hierarchy of triggers to avoid was established after discussion with the therapist (Table 1). From session 3 to 8 , each of these eight triggers was desensitized until the LOU decreased to zero, starting with T1 (the lowest LOU) and ending with T8 (the highest). As the sessions progressed, the patient gradually gained control of his fear and anxiety about public urination. After the fifth session, he voluntarily exposed himself to a situation addressed in the previous session, although he had not been given any suggestions or homework about in vivo exposure. At the final session, he reported that he was able to visit and use any public restroom.

\section{DISCUSSION}

We report the successful application of DeTUR, an addiction protocol of EMDR, in a person with chronic paruresis that had lasted more than one decade despite previous pharmacotherapy and cognitive behavioral therapy. After treatment, the patient reported reduction of the symptoms to nonclinical levels base on three self-report measures covering the domains of social phobia (i.e., general and social anxiety, avoidance, and fear of negative evaluation); he no longer met the diagnostic criteria for social anxiety disorder, and more importantly, he was able to use public restrooms. This complete remission was maintained at the one-year follow-up.

Table 1. The hierarchy of triggers causing phobic avoidance in a patient with paruresis

\begin{tabular}{clc}
\hline Triggers & & Urge to avoid (0-0) \\
\hline T1 & A small restroom for one person in a pub & 3 \\
T2 & A familiar restroom with one or two others present & 4 \\
T3 & A restroom in a department store with individual partitions in the presence of four others & 5 \\
T4 & A quiet restroom in a subway station, with others present, but no one waiting & 6 \\
T5 & A small restroom with two urinals & 7 \\
T6 & A small restroom full of people, some waiting & 8 \\
T7 & A college restroom between lectures, very busy and full of people & 9 \\
T8 & A public toilet with portable urinals such as at a camping site, very compact and full of people & 10 \\
\hline
\end{tabular}


The patient's improvement is not likely to be due to a placebo effect because during the course of his illness, he had already received pharmacotherapy and cognitive behavioral therapy without improvement, although it is not clear whether the doses and lengths of these treatments were adequate. There is a possibility that the voluntary in vivo exposure to which the patient submitted himself affected the outcome because such exposure has been considered a treatment of choice for paruresis. ${ }^{3}$ However, it was not carefully planned or repeated but rather was a one-time self-test of a situation he had dealt with successfully in the sessions.

The significance of this case is that it suggests that DeTUR may reduce social anxiety. As stated above, DeTUR targets an urge and its triggers, and there have been anecdotal reports that it can be extended to urge-related disorders such as eating disorders, obsessive compulsive disorders, anger outbursts, and also possibly to anxiety or phobia. ${ }^{12}$

DeTUR differs from EMDR in that it reprocesses present triggers, rather than past memories, but the patient may in fact recall and desensitize memories of phobia because each triggering situation reflects a previous representative memory. It is not clear at present whether DeTUR is superior to standard EMDR for phobia in terms of effectiveness and efficiency. Hence, a study is needed comparing standard EMDR and DeTUR for phobia.

The limitations of the present study include its design as a case report, the possible effect of the self-exposure on the treatment outcome, and the use of self-report measurement instruments. In addition, we should mention that the patient was well-motivated (treatment seeking) and had no other psychiatric comorbidities. CBT is considered the psychological treatment of choice for SAD; however, as a recently published clinical trial noted, only $36 \%$ of those who received CBT achieved remission and $40 \%$ did not respond to the treatment. ${ }^{13}$ The present case report suggests that DeTUR might be a useful treatment option for paruresis. It had a positive effect within a relatively short period of time and there was no need for homework. Additional research is required to extend this finding and confirm DeTUR as a treatment modality for SAD.

\section{REFERENCES}

1. American Psychiatric Association. Diagnostic and Statistical Manual of Mental Disorders, Fifth Edition. Arlington, VA: American Psychiatric Association; 2014.

2. Bohn P, Sternbach H. Current knowledge and research directions in the treatment of paruresis. Depress Anxiety 1997;5:41-42.

3. Boschen MJ. Paruresis (psychogenic inhibition of micturition): cognitive behavioral formulation and treatment. Depress Anxiety 2008;25: 903-912.

4. Soifer S, Himle J, Walsh K. Paruresis (shy bladder syndrome): a cognitive-behavioral treatment approach. Soc Work Health Care 2010;49: 494-507.

5. De Jongh A, Ten Broeke E, Renssen MR. Treatment of specific phobias with Eye Movement Desensitization and Reprocessing (EMDR): protocol, empirical status, and conceptual issues. J Anxiety Disord 1999; 13:69-85.

6. Faretta E. EMDR and cognitive-behavioural therapy in the treatment of panic disorder: a comparison. Riv Psichiatr 2012;47(2 Suppl):19-25.

7. Gauveau P, Bouchard S. Preliminary evidence for efficacy of EMDR in treating generalized anxiety disorder. J EMDR Pract Res 2008;2:26-40.

8. Popky AJ. The Desensitization of Triggers and Urge Reprocessing (DeTUR) Protocol. Eye Movement Desensitization and Reprocessing (EMDR) Scripted Protocols: Special Populations. New York: Springer Publishing; 2010.

9. Amir N, Foa EB, Coles ME. Automatic activation and strategic avoidance of threat-relevant information in social phobia. J Abnorm Psychol 1998;107:285-290.

10. Spielberger C, Gorsuch R, Lushene R. Manual for the Trait-State Anxiety Inventory. Palo Alto, CA: Consulting Psychologists; 1970.

11. Watson D, Friend R. Measurement of social-evaluative anxiety. J Consult Clin Psychol 1969;33:448-457.

12. Popky AJ. DeTUR, an Urge Reduction Protocol for Addictions and Dysfunctional Behaviors. In: Shapiro R, Editor. EMDR Solutions: Pathways to Healing. New York: W.W. Notron \& Company, Inc; 2005.

13. Leichsenring F, Salzer S, Beutel ME, Herpertz S, Hiller W, Hoyer J, et al. Psychodynamic therapy and cognitive-behavioral therapy in social anxiety disorder: a multicenter randomized controlled trial. Am J Psychiatry 2013;170:759-767. 